\title{
Editorial
}

\section{Acceso abierto y sistemas de evaluación de la ciencia, ¿calidad invisibilizada vs cantidad comercializada?}

Referirnos al acceso abierto en Latinoamérica es involucrar el término de "ciencia abierta” y analizar posibles sistemas de evaluación de la ciencia de calidad, contrapuestos al sistema de evaluación internacional y globalizado de la corriente primaria, "mainstream" [Fernanda Beigel, 1]. A continuación, se describe una visión generalizada sobre la ruta desde el acceso abierto y los sistemas de evaluación, en el contexto de diversas exposiciones desde la UNESCO, CLASO, Fundación Carolina, CEPAL, entre otros; en vías de una transformación y avance hacia la ciencia abierta.

Una ciencia abierta debe considerar el derecho humano a participar en la ciencia y a beneficiarse de ella, a leer y publicar [Dominique Babini, 2]. Por lo que definir ciencia abierta es muy complejo, a la fecha aún no se consolida este concepto. Desde espacios como la UNESCO, CEPAL, ForoLAC 2018 [3] y diversas declaraciones de comunidades latinoamericanas, se ha propuesto la ciencia abierta como una ciencia inclusiva y de apertura a la diversidad del conocimiento; donde domine el acceso, datos, software y evaluaciones abiertas, con recursos e incentivos abiertos, respaldados por infraestructuras y repositorios fortalecidos; referenciados a un circuito o sistema transnacional en acceso abierto no comercial [ 1].

Por su parte, el circuito mainstream o corriente principal, creada en la década de los 50 del siglo XX, cuando a la par del conflicto bélico de la Segunda Guerra Mundial, se da un auge de la actividad científica y tecnológica, y se consolidan las editoriales científicas en la demanda de revistas científicas de prestigio; tercerizando y comercializando la producción científica hasta nuestros días, protagonizado por el "factor de impacto"; creado en la misma década, y evolucionando hasta nuestro días (SCI, JCR, SCOPUS, etc.) [1]. Dicho sistema ha actuado como un oligopolio desde entonces, utilizando el factor de impacto como criterio de calidad, que mide cantidad de citas en periodos de tiempo preestablecidos, sin sopesar la naturaleza, campo de los trabajos, ni contribuciones del mismo. Sin embargo, este criterio "erróneamente" considerado como calidad de la producción científica, se terminó de consolidar en la década anterior (2000-2010), al sumarse para generar los rankings universitarios, que dieron inicio a una competencia entre universidades en todos los continentes [1], dejando a un lado la identidad regional y sus implicaciones. Los sistemas de evaluación de la corriente principal se consolidaron como la ciencia internacional y global, como la "verdadera ciencia", totalmente opuesto a lo que se construía desde Latinoamérica como una ciencia periférica, no jerárquica, endogámica y local [1].

Por otra parte, Latinoamérica daba la apertura y el gran aporte para el mundo, sobre el tema del acceso abierto y la ciencia abierta. Nuestra región, ha privilegiado políticas y modalidades del acceso abierto que son colaborativas, financiadas con fondos públicos, con fuerte protagonismo de las universidades y organismos de ciencia gubernamentales, con políticas científicas sin fines comerciales, gestionados como un bien común. Esa forma de implementarlo nos distingue, y se encuentra destacada en diversas reuniones internacionales. Este modelo con protagonismo de organismos científicos sin fines de lucro y sin intermediación comercial, genera tensión con un modelo internacional que avanza en la privatización de la producción y comunicación científica, que comercializa no solo con la publicación de revistas desde la corriente principal, sino con indicadores de evaluación [2]. Desde hace 20 años, se consolidaron estos lineamientos mediante la creación temprana de portales de revistas científicas en la región iberoamericana, para la visibilidad en acceso abierto e indicadores para las revistas de calidad de Latinoamérica (Scielo, Latindex, Redalic, Amelica, Dialnet, Redib) [2].

La evaluación científica en nuestra región, no obstante, presenta un problema complejo, basado en que el factor de impacto implica un tipo de cuantificación y generación de ranking internacional, donde las revistas son medidas, dominando así la voluntad y el incentivo de los investigadores latinoamericanos a publicar en revistas de fuera de Latinoamérica, ya que las mediciones y recompensas salariales y de clasificación en sus instituciones, están referidas a los sistemas de evaluación tradicional (mainstream), respecto al factor de impacto [1]. Es por ello, que el camino desde el acceso abierto hacia la ciencia abierta, refiere desafíos para la transición a la ciencia abierta en Iberoamérica, que atraviesan desde los riesgos de la ciencia abierta comercial (con APC y suscripciones) y la necesidad de indicadores relevantes para la evaluación de la calidad e incentivos en acceso abierto, que logren estimular y valorizar la divulgación regional de la ciencia local, nacional y regional, revalorizando nuestras revistas nacionales [1].

De aquí, que en este año 2020 con los diversos y nuevos escenarios mundiales debido a la pandemia por Covid-19, se contribuyó a un doble movimiento fuerte y acelerado, respecto a la evaluación de la ciencia para incluir la valoración de la ciencia abierta en los procesos de evaluación de la investigación, así como su revisión, en miras de una reforma de la evaluación académica responsable, a una integridad de la evaluación en investigación, la redefinición del concepto de excelencia o calidad de investigación, y por último, hacia la reflexión en la necesidad de alejarnos de la dependencia de 


\section{Editorial}

las métricas globalizadas, para explorar otros modos de evaluación de la ciencia, manifestados y suscritos en diversas declaraciones [Laura Rovelli, 2]. Una de ellas, la Declaración de San Francisco DORA (Fundación Carolina), donde el proceso de evaluación ligado a la actividad científico-académica, está basado en el progreso y transferencia del conocimiento, valorando las contribuciones y aportaciones según su relevancia o valor intrínseco para el progreso de cada campo, y la transferencia del conocimiento como una doble vía. Este año 2020, colocó aún más la ciencia abierta y la necesidad de hacer ciencia abierta en la palestra, demostrado a través del rápido avance en la investigación y desarrollo de vacunas, ya que de manera excepcional, las grandes editoriales y repositorios han abierto la producción científica y la han hecho accesible, solo en este escenario de pandemia [José Antonio Sanahuja, 2]. De allí la necesidad de avanzar en métricas complementarias y sistemas de evaluación, que valoren la ciencia en acceso abierto publicada en nuestros países [Laura Rovelli, 2].

Finalmente, las actuales discusiones se centran en que: "la ciencia abierta debe ser un factor de crecimiento para toda nuestra región, incluyendo a todos los sistemas científicos grandes y pequeños, todos de manera igualitaria, inclusiva y con equidad, para el progreso científico desde adentro, y garantizando que el beneficio sea para todos; considerando que el derecho al conocimiento y a la ciencia, es un derecho humano" [Lidia Brito, 2]. Desde esta óptica, claramente inclusiva, se hace indispensable aplicar políticas y lineamientos que nos acerquen a los sistemas de evaluación globalizados ya establecidos (mainstream), para que no se genere un mayor distanciamiento y desigualdad de la producción científica latinoamericana.

En esta oportunidad, se presenta para el volumen 44 número 1 año 2021, un compendio de siete artículos científicos, insertos en las áreas de la ingeniería química (biomateriales y alimentos), ingeniería industrial (gerencia y gestión de calidad), ingeniería civil (materiales de construcción y tecnología del hormigón) y ciencias básicas en computación; con autores y coautores de diversas filiales institucionales y nacionalidades: siete instituciones universitarias en Ecuador, dos en Perú, Cuba y Venezuela, y una en Chile y España. Entre ellas están: Universidad Laica Eloy Alfaro de Manabí (Ecuador), Universidad Técnica de Machala (Ecuador), Universidad Nacional de Educación (Ecuador), Universidad Nacional de Chimborazo (Ecuador), Universidad Yachay Tech (Ecuador), Universidad Técnica de Manabí (Ecuador),Universidad Nacional Toribio Rodríguez de Mendoza de Amazonas (Perú), Universidad Nacional Agraria La Molina (Perú), Universidad de Córdoba (España), Universidad de Valparaíso (Chile), Universidad Tecnológica de La Habana "José Antonio Echeverría” (Cuba), Universidad de Sancti Spíritus “José Martí Pérez" (Cuba), Universidad Simón Bolívar (Venezuela) y Universidad del Zulia (Venezuela). Una nueva entrega que demuestra una vez más, el arduo y honorable trabajo de autores, revisores, editores científicos, y comité editorial; permitiendo la visibilización de productos científicos inéditos y de calidad. Gracias a todos, y un muy venturoso y feliz nuevo año 2021.

Profesora Dra. Valentina Millano-González Directora Revista Técnica de la Facultad de Ingeniería

[1] Ciencia abierta y sistemas de evaluación de la ciencia. Fernanda Beigel presidenta del Comité Asesor de la UNESCO para Ciencia Abierta. Ciclo de Webinars sobre Ciencia Abierta 2020 organizados por la Biblioteca de la CEPAL. https://www.cepal. org/es/notas/ciencia-abierta-sistemas-evaluacion-la-ciencia.

[2] Presentación del libro "Tendencias Recientes en las Políticas Científicas de Ciencia Abierta y Acceso Abierto en Iberoamérica”. CLASO y Fundación Carolina. Diciembre 2020. https://www.clacso.org/actividad/presentacion-del-librotendencias-recientes-en-las-politicas-cientificas-de-ciencia-abierta-y-acceso-abierto-en-iberoamerica/.

[3] Declaración de Panamá 2018, Foro Ministerial para el Desarrollo en América Latina y el Caribe 10a edición, FOLAC, undp. org/forolac, https://www.cepal.org/es/comunicados/declaracion-panama-reafirma-compromiso-autoridades-la-regionreducir-desigualdades.

[4] Declaración de San Francisco sobre la evaluación de la investigación DORA. Fundación Carolina. 2012. http://dx.doi. org/10.14201/orl.17845. https://sfdora.org/read/read-the-declaration-espanol/. 